\title{
Gravitational collapse and structure formation in an expanding universe with dark energy
}

\author{
Manvendra Pratap Rajvanshi, Tuneer Chakraborty \& J. S. Bagla
}

Observations show that the expansion of the Universe is accelerating. This requires that the dominant constituent of matter in the Universe has some unusual properties like negative pressure. This exotic component has been given the name dark energy. We work with the simplest model of dark energy, the cosmological constant introduced by Einstein. We study the evolution of spherical over-densities in such a model and show that there is a minimum over-density required for collapse: perturbations with a smaller amplitude do not collapse. This threshold is interesting as even perturbations with a positive over-density and negative energy do not collapse in finite time. Further, we show that perturbations with an amplitude larger than, but comparable to the threshold value, take a very long time to collapse. We compare the solutions with the case when dark energy is absent.

\section{Introduction}

Ever since Newton formulated his theory of gravitation, there has been a quest for a mathematical theory for large-scale dynamics of the Universe. At cosmological scales, gravity is the dominant force, hence the theory of gravitation is central to study of Universe. The early discussion in this context focused on the question of stability of the large-scale distribution of matter, and whether the Universe is required to be infinite in extent for stability [1].

Present day models of the Universe are based on relativistic theories of gravity. Einstein proposed a static cosmological model soon after proposing the general theory of relativity (see [2] as well as [3] and references therein). He invoked the cosmological term, now known as the cosmological constant in order to obtain

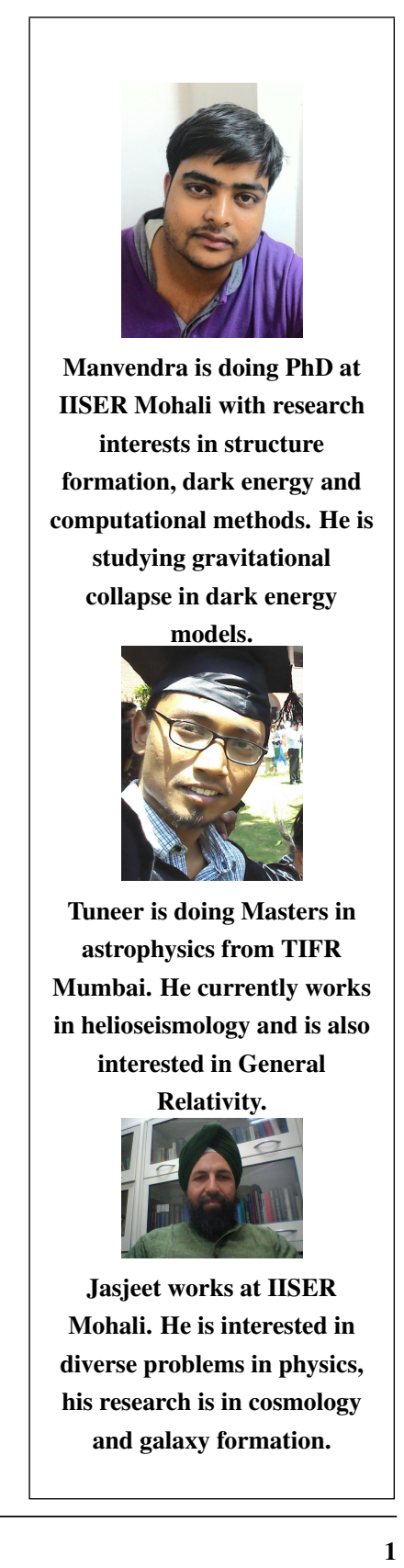


a static model. It was soon shown that the static model is unstable to perturbations, i.e., if there are any fluctuations in density then the under-dense regions expand and over-dense regions undergo rapid collapse leading the universe away from the static equilibrium.

Over the next fifteen years, many scientists tried to construct relativistic cosmological models. Friedmann [4, 7], Lemaitre [5, 8], Robertson [6, 9] and Walker [10] arrived at the simplest relativistic cosmological models. These models assumed a universe that is homogeneous and isotropic. This assumption was an extension of the Copernican principle that there is no preferred location or direction in the Universe. The models can be described as different manifestations of a space-time metric that is now called the Friedmann-Lemaitre-Robertson-Walker (FLRW) metric. A common feature of solutions in all the models was an expanding universe, which was shown to match observations made by Slipher [11], and, Hubble [12] and Humason. In any region, it is expected that galaxies are seen to move away from each other due to expansion, and the recession velocities are larger for galaxies at a higher distance.

$$
V=H_{0} R
$$

where $R$ is the distance from an observer to a galaxy, $V$ is the recession speed, and $H_{0}$ is the Hubble's constant and its observed value is close to $70 \mathrm{~km} \cdot \mathrm{s}^{-1} \cdot \mathrm{Mpc}^{-1}$. This relation is valid for distances that are much smaller than $c / H_{0} \simeq 4286 \mathrm{Mpc}$. Here Mega Parsec $(\mathrm{Mpc})$ is a unit of distance and equals $3.08 \times 10^{22} \mathrm{~m}$. The Hubble's constant is dimensional and also gives us a natural time scale of $\sim 1.4 \times 10^{10}$ years. For more details about the Hubble's law and its discovery, see [14].

The inclusion of a cosmological constant in the equations describing the Universe leads to interesting consequences. A cosmological constant can lead to accelerated expansion of the Universe. The cosmological constant has been used at many times to alleviate the age problem: without the cosmological constant the age of the Universe is constrained to be smaller than $\tau_{0}=H_{0}^{-1}$, where $H_{0}$ is the Hubble's constant. Earliest measurements of the Hub- 
ble's constant were incorrect and yielded $\tau_{0} \simeq 1.8$ billion years (with $H_{0}=550 \mathrm{~km} \cdot \mathrm{s}^{-1} \cdot \mathrm{Mpc}^{-1}$ ), smaller than the age of the oldest rocks on the Earth1. The measurements of the Hubble's constant were corrected over three decades when several subtle problems with the original measurements were discovered and corrections were made. For example, the differences between population I and population II stars were realized by Baade [13]. Population I stars are similar to the Sun in their chemical composition. Population II stars have a much smaller amount of elements other than Hydrogen and Helium. Astronomers refer to all other elements as metals and the metallicity of population II stars is much lower than that of population I stars. Population II stars are found in the halo of the Galaxy. Baade discovered that Cepheid variables in the two populations [13] have a different period luminosity relation and this affects the distance estimates. Further, it was realized that Hubble and Humason had confused HII regions with bright stars and this led to an incorrect inference of distance. It took another four decades for the error bars to shrink below $10 \%$, and by this time it had been shown using observations of supernovae type $\mathrm{Ia}^{2}$ that the cosmological constant is non-zero and dominates the energy budget of the Universe[15, 16, 17]. Observations of supernovae of type Ia indicate that the expansion of the Universe is accelerating, and the cosmological constant is the simplest model that fits all available observations. The general term used for the component responsible for an accelerating universe is dark energy, and the cosmological constant discussed here is the simplest model of dark energy. As we will see below, dark energy is required to have negative pressure.

In this paper we present a discussion of the effect of a cosmological constant on non-linear evolution of density perturbations. Dynamics in the cosmological model called $\Lambda \mathrm{CDM}$, which incorporates both cold dark matter ${ }^{3}$ and dark energy in form of cosmological constant has been studied in detail. It is possible to study non-linear collapse of perturbations analytically if we re-

\footnotetext{
${ }^{1}$ See https://en.wikipedia.org/wiki/Age_of_the_Earth for discussion of the determination of the age of oldest rocks by Arthur Holmes.
}

RESONANCE | month year
${ }^{2}$ Supernovae of type Ia are very bright. These events mark the crossing of the Chandrasekhar mass limit for a white dwarf as it gains mass from a companion star. The luminosity of supernovae is related to the decline of flux in fifteen days. This permits astronomers to estimate the distance and redshift for these objects independently, and hence constrain the rate of expansion.

\footnotetext{
${ }^{3}$ Cold dark matter (CDM) is a class of dark matter where the velocity dispersion of dark matter particles is very small, and bulk velocities are also nonrelativistic. Observations indicate that most of the dark matter is of this type.
} 
strict ourselves by imposing a symmetry, e.g., spherical symmetry on the perturbations. We assume that the density perturbation is spherically symmetric, all bulk motions are purely radial, and the matter undergoing collapse does not have a significant velocity dispersion. Collapse of perturbations in such a case was first studied by Gunn and Gott[18] and this has been reviewed by us in an earlier article [19]. We strongly urge the reader to refer to that article first and treat this article as part II in the series. The analysis for spherical collapse has been generalized to the case where a cosmological constant is present [21, 22, 23, 24]. We follow the approach proposed by Barrow and Saich [23] in this paper. Basic equations describing the Universe are introduced in $\S 2$, these are modified to describe spherically symmetric perturbations in $\S 3$ where we also discuss initial conditions. Presence of a cosmological constant leads to a critical threshold for the initial density contrast: smaller density perturbations do not collapse. This is discussed in section $\S 3$ and $\S 4$. Detailed evolution of perturbations is discussed in $\S 4$ and $\S 5$.

\section{2. $\Lambda$ in FLRW Equations}

Einstein's theory of general relativity provides the framework for studying dynamics of the Universe. This is also the appropriate framework for studying evolution of large-scale perturbations. However, in the context of spherically symmetric perturbations and only non-relativistic matter other than the cosmological constant, the general relativistic equations have a well defined Newtonian limit. The usual force equation is modified with the addition of a repulsive interaction term. Following [19], we write the equation of motion for a thin spherical shell at a distance $R$ from the centre. The choice of origin is arbitrary in case of a smooth universe.

$$
\ddot{R}=-\frac{G M}{R^{2}}+\frac{\Lambda}{3} R
$$

The first term on the right represents gravitational attraction due to the mass enclosed in the shell, and the second term represents the repulsion due to the cosmological constant $\Lambda$. The cosmolog- 
ical constant does not depend on location in space or on time. We can calculate the first integral from equation (1) with the assumption that the mass $M$ within the shell is a constant:

$$
\frac{1}{2} \dot{R}^{2}-\frac{G M}{R}-\frac{1}{6} \Lambda R^{2}=\text { constant }=\alpha
$$

The constant here plays the role analogous to energy in particle dynamics. The shell may expand or contract, all other modes of motion lead to departures from homogeneity and isotropy ${ }^{4}$ and hence are not admissible in this model. Using comoving coordinates $\mathbf{r}$ (see, e.g., [19]) $\mathbf{R}(t)=a(t) \mathbf{r}$, where the physical coordinate $\mathbf{R}$ may change with time but $\mathbf{r}$ remains fixed for fundamental observers in an expanding/contracting homogeneous and isotropic universe, i.e., there is a group of observers whose only motion in physical coordinates is expansion or contraction and this can be described entirely in terms of an overall expansion or contraction of the Universe with no other component to their motion. The expansion and contraction is described by the scale factor $a(t)$.

By expressing the mass $M$ in terms of the average matter density $\bar{\varrho}$, and changing over from physical coordinate $\mathbf{R}$ to comoving coordinate $\mathbf{r}$, equations (1-2) can be written as:

$$
\begin{aligned}
\frac{\ddot{a}}{a} & =-\frac{4}{3} \pi G \bar{\varrho}+\frac{1}{3} \Lambda \\
\left(\frac{\dot{a}}{a}\right)^{2}+\frac{k}{a^{2}} & =\frac{8 \pi G}{3} \bar{\varrho}+\frac{1}{3} \Lambda
\end{aligned}
$$

Here, $k$ is related to the constant $\alpha$ in equation (2). By convention, it takes values $0, \pm 1$. A comparison with the equations in [19] shows that the cosmological constant counters deceleration in expansion due to matter. Matter density is diluted during expansion $^{5}$, however the cosmological term is a constant and its effect does not suffer a corresponding reduction. Thus we expect the cosmological constant to play an important role at late times in an expanding universe as it can overtake matter as the dominant source in Friedmann equations.

Of special interest here is a spatially flat universe $(k=0)$ as this is consistent with observations [20]. In this case, the Friedmann

\footnotetext{
${ }^{4}$ Homogeneity is the translation symmetry, implying that the distribution of matter is the same everywhere at a given time. Isotropy is the invariance under rotation, i.e., the matter distribution is the same in all directions.
} 
equation takes the form:

$$
H^{2}=\left(\frac{\dot{a}}{a}\right)^{2}=\frac{8 \pi G}{3} \bar{\varrho}+\frac{1}{3} \Lambda=H_{0}^{2}\left[\Omega_{M}\left(\frac{a_{0}}{a}\right)^{3}+\Omega_{\Lambda}\right]
$$

${ }^{6}$ Density parameter is the density in units of the critical density, see [19] or any textbook on cosmology for a complete definition.
Here, $a_{0}$ is the present value of the scale factor, $H_{0}$ is the present value of the Hubble parameter $H=\dot{a} / a, \Omega_{M}$ and $\Omega_{\Lambda}$ are the density parameters for matter and cosmological constant, respectively $^{6}$. For a flat universe, we have $\Omega_{M}+\Omega_{\Lambda}=1$. The solution to this equation gives the scale factor as a function of time:

$$
\left(\frac{a}{a_{0}}\right)^{3}=\frac{\Omega_{M}}{\Omega_{\Lambda}} \sinh ^{2}\left(\frac{3 t H_{0} \sqrt{\Omega_{\Lambda}}}{2}\right)
$$

When $t H_{0} \ll 1$, i.e., at early times, this matches with the evolution of scale factor for a matter dominated universe with $a(t) \propto$ $t^{2 / 3}$. At late times, $t H_{0} \gg 1$, we get accelerated expansion with $a(t) \propto \exp \left(2 t H_{0} \sqrt{\Omega_{\Lambda}}\right)$. The rate of expansion begins to accelerate when $\ddot{a}>0$, i.e.,

$$
\begin{aligned}
-\frac{4}{3} \pi G \bar{\varrho}+\frac{1}{3} \Lambda & =-\frac{1}{2} H_{0}^{2} \Omega_{M}\left(\frac{a_{0}}{a}\right)^{3}+H_{0}^{2} \Omega_{\Lambda}>0 \\
\Longrightarrow \frac{a}{a_{0}} & >\left(\frac{\Omega_{M}}{2 \Omega_{\Lambda}}\right)^{1 / 3}
\end{aligned}
$$

In our Universe, the transition to accelerating expansion happened when the scale factor was slightly less than $60 \%$ of its present value. Note that this epoch is distinct from the one where we transition from a matter dominated to a dark energy dominated universe: this transition happens when the scale factor is close to $75 \%$ of its present value. Thus accelerated expansion starts well before the energy density in the Universe is dominated by dark energy.

\section{Spherical Over-density}

We can consider a spherical over-density in a flat universe $(k=$ $0)$. We consider a shell with radius $R$. Due to the over-density, the shell does not correspond to a fundamental observer and its comoving coordinates change in time, i.e., it will depart from the 
fundamental observer located at the same radius at the initial time. The shell contains mass $M$ and average density $\varrho=(1+\bar{\delta}) \bar{\varrho}$, where $\bar{\varrho}$ is the average density of the Universe as defined earlier and $\bar{\delta}$ is the average density contrast inside the shell. We choose initial conditions such that $R_{\text {in }} \propto a_{\text {in }}$ and the shell is comoving with the Hubble expansion, e.g., $\dot{R}_{i n}=H_{i n} R_{i n}$. This, along with the Friedmann Equation for the background universe allows us to compute the constant in equation (2).

$\alpha=\frac{1}{2} \dot{R}_{i n}^{2}-\frac{G M}{R_{\text {in }}}-\frac{1}{6} \Lambda R_{\text {in }}^{2}=-\frac{1}{2} H_{i n}^{2} \Omega_{M}\left(a_{i n}\right) R_{\text {in }}^{2} \bar{\delta}_{\text {in }} \simeq-\frac{1}{2} H_{i n}^{2} R_{\text {in }}^{2} \bar{\delta}_{\text {in }}$

Here, $\Omega_{M}\left(a_{i n}\right)$ is the density parameter for matter at the initial epoch. The only approximation that has been made here in the last step is that the initial conditions are set at an epoch where matter dominates over dark energy, i.e., $\Omega_{M}\left(a_{i n}\right) \simeq 1$. If the region is over-dense then the constant is negative, however this does not necessarily mean that the shell will undergo collapse. To see this, we consider the condition for the expanding shell to decelerate to rest and begin collapsing, which means that $\dot{R}$ must go to zero.

$$
-\frac{G M}{R}-\frac{1}{6} \Lambda R^{2}=-\frac{1}{2} H_{\text {in }}^{2} R_{\text {in }}^{2} \bar{\delta}_{\text {in }}
$$

This is a cubic equation in $R$ and it has a real and positive solution only if the value of $\bar{\delta}_{\text {in }}$ is above a certain threshold value $\delta_{t h r}$ for a given set of cosmological parameters. The threshold density contrast is independent of the scale of the perturbation. We can express $\delta_{t h r}$ as the solution to the following equation:

$$
27 \Omega_{\Lambda}\left(1+\delta_{t h r}\right)^{2} a_{i n}^{3}-4 \Omega_{M}\left(\delta_{t h r} a_{0}\right)^{3}=0
$$

If we set up initial conditions at $a_{0} / a_{\text {in }}=100$ then $\delta_{t h r} \simeq 0.02$ for $\Omega_{\Lambda}=0.7$. Perturbations with $0<\bar{\delta}_{\text {in }} \leq \delta_{\text {thr }}$ are over dense but will never collapse to form a halo.

The critical threshold for density contrast obtained here is very different in character to a similar threshold obtained in an open model ( $k=-1$ in equation (4)) in that the constant $\alpha$ is negative for all positive $\bar{\delta}_{i n}$ for the models under consideration. As $\alpha$ is related to the energy of the shell, this seems to indicate that the 
shell is bound even though it does not collapse in finite time. In an open model for the Universe, the threshold density contrast corresponds to $\alpha=0$, implying that perturbations with a lower density contrast are not bound.

It is important to note that even for a closed model, the same criterion leads to a threshold, except that in this case the threshold is a finite negative value for $\bar{\delta}_{\text {in }}$ implying that even some underdense regions may undergo collapse.

Thus there are two regimes for perturbations that do not collapse in presence of dark energy:

- Energy of the perturbation is non-negative. This is true only if the density contrast is negative. The perturbation in this case is clearly not bound and will not collapse.

- Energy of the perturbation is negative, but the density contrast is less than or equal to the threshold value required for collapse in finite time, i.e., $0 \leq \bar{\delta}_{i n} \leq \delta_{t h r}$. In this case the perturbation is bound (negative energy) but will not collapse in finite time. In this case dark energy dominates the evolution of the perturbation.

This is to be contrasted with the open models where the energy is non-negative only if the density contrast is positive and greater than zero, and such perturbations do not collapse.

\subsection{Equations and Solutions}

Initial conditions allow us to obtain the following equation for dynamics of a shell

$$
\dot{R}^{2}=\Omega_{\Lambda} R^{2}+\Omega_{M}\left(\frac{a_{0}}{a_{i n}}\right)^{3} \frac{\left(1+\bar{\delta}_{i n}\right) R_{\text {in }}^{3}}{R}-\Omega_{M}\left(\frac{a_{0}}{a_{i n}}\right)^{3} \delta_{i n} R_{i n}^{2}
$$

where $\delta_{\text {in }}$ is initial density contrast and dot represents derivative with respect to $t H_{0}$, with $H_{0}$ being the Hubble's constant. At any time $t$ the density contrast can be obtained from following relation:

$$
(1+\bar{\delta})=\left(1+\bar{\delta}_{\text {in }}\right)\left(\frac{a}{a_{\text {in }}}\right)^{3}\left(\frac{R_{\text {in }}}{R}\right)^{3}
$$




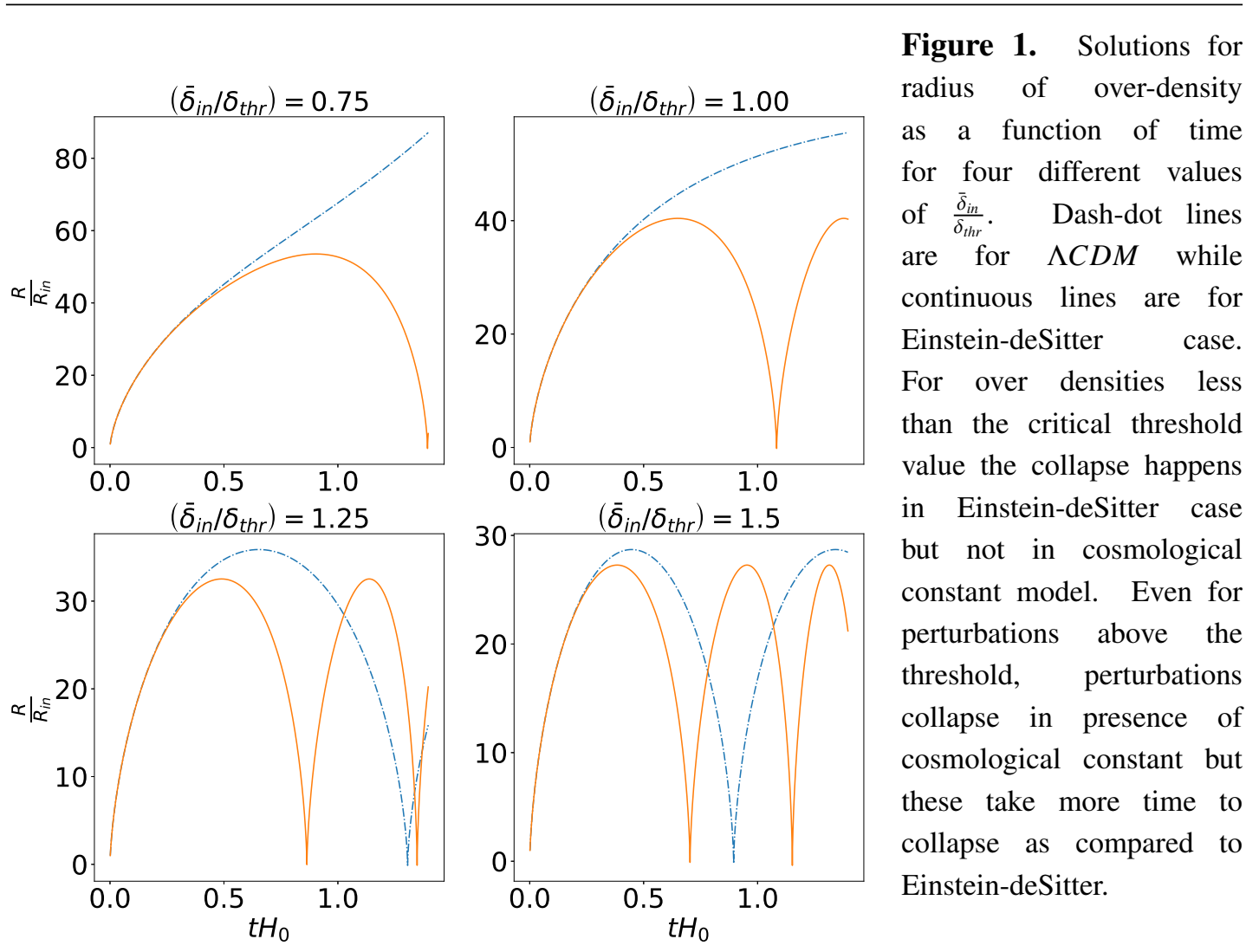

This relation follows from conservation of mass within the shell. The factor $1+\bar{\delta}_{\text {in }}$ accounts for the initial over density within the shell, $\left(a_{i n} / a\right)^{3}$ is the evolution of the average density of the universe that is the background in which the over density is evolving, and $\left(R_{i n} / R\right)^{3}$ is the change in density of the shell due to change in its radius.

Solution to equation $(\sqrt{10})$ can be obtained in the form of an integral (Hyper-geometric function) which has to be evaluated numerically. Instead one can also solve the differential equation (10p) numerically and obtain the solution. Here we present the solution and contrast it with solution when there is no $\Lambda$, i.e., with the Einstein-deSitter case.

We plot solutions of equation (10) in Figure 1. In this figure 
we compare the solutions with the same initial conditions for a universe with cosmological constant with the corresponding solutions in the Einstein-deSitter universe. We have plotted four cases around $\bar{\delta}_{\text {in }} / \delta_{t h r}=1$, the threshold initial value that divides collapsing and non-collapsing solutions. We find that in the Einstein-deSitter universe, all over-densities lead to collapse whereas in presence of cosmological constant, only regions with $\bar{\delta}_{i n}>\delta_{t h r}$ lead to collapse. Further, in case where cosmological constant is present, collapse takes much longer time than the corresponding over-density in an Einstein-deSitter universe. The difference between the two diminishes as we go to $\bar{\delta}_{i n} / \delta_{t h r} \gg 1$. We also note that the radius at which the shell begins to recollapse is larger in the presence of dark energy.

\section{Turn Around}

The initial conditions are set such that the shell is expanding with the Universe. For the shell to collapse and form a bound structure, the expansion of the shell must come to a halt and the radial velocity must vanish at a finite time. This stage of zero radial velocity is called turn around where the perturbation reaches its maximum radius. Whether we reach such a stage or not depends on the competition between attractive gravitational force and repulsion due to cosmological constant. Setting $d R / d\left(t H_{0}\right)=0$ in equation $([10)$ ) we obtain an expression for turn around radius by solving the equation:

$$
\begin{aligned}
R_{t a}= & \frac{3\left(1+\bar{\delta}_{i n}\right)}{\bar{\delta}_{i n}} R_{i n}\left(\frac{4 \Omega_{M}\left(\bar{\delta}_{i n} a_{0}\right)^{3}}{27 \Omega_{\Lambda}\left(1+\bar{\delta}_{i n}\right)^{2} a_{i n}^{3}}\right)^{1 / 2} \\
& \sin \left[\frac{1}{3} \arcsin \left\{\left(\frac{27 \Omega_{\Lambda}\left(1+\bar{\delta}_{i n}\right)^{2} a_{i n}^{3}}{4 \Omega_{M}\left(\bar{\delta}_{i n} a_{0}\right)^{3}}\right)^{1 / 2}\right\}\right]
\end{aligned}
$$

For the solution to be physically meaningful, the argument of arcsin in curly brackets must be less than unity. This is the origin of the condition given in equation (9) that the initial density contrast must be larger than the threshold value $\delta_{t h r}$ for the perturbation to reach turn around. 


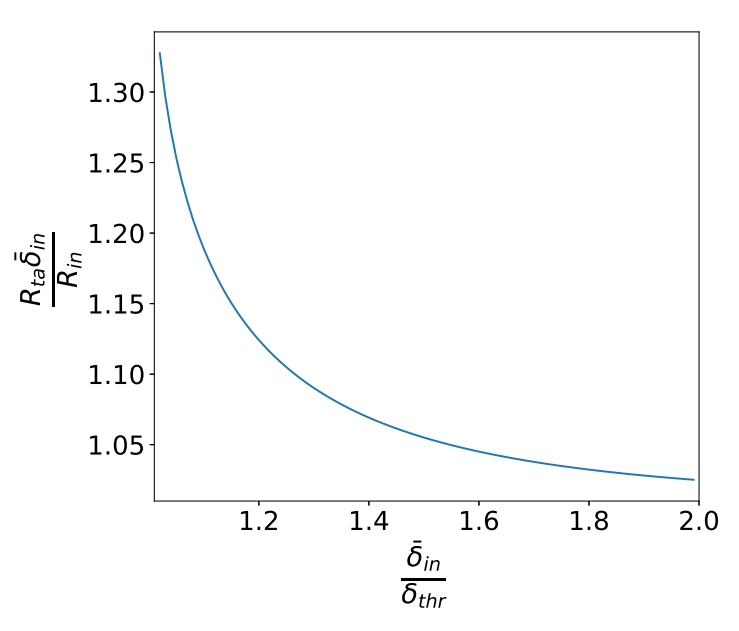

\subsection{Characteristics at turn around}

The radius of the perturbation at turn around is fixed uniquely by the initial over-density. In case of the Einstein-deSitter universe, the radius of the shell increases by a factor of $1 / \bar{\delta}_{\text {in }}$ from the initial value, thus $R_{t a}=R_{i n} / \bar{\delta}_{i n}$. Thus a perturbation with a larger initial over-density expands by a smaller factor before turning around and collapsing. In Figure 2 we show the combination $R_{t a} \bar{\delta}_{i n} / R_{i n}$, which is unity in the Einstein-deSitter universe. We plot this combination as a function of $\bar{\delta}_{i n} / \delta_{t h r}$. We find that $R_{t a} \bar{\delta}_{i n} / R_{i n}$ tends to unity as $\bar{\delta}_{i n} / \delta_{t h r}$ becomes much larger than unity. However, as we approach the threshold value $\bar{\delta}_{i n} / \delta_{t h r}=1$ from above, the turn around radius becomes larger than the corresponding perturbation in the Einstein-deSitter universe. We can conclude from here that the density of the perturbation at turn around is lower than the corresponding perturbation in the Einstein-deSitter universe.

In Figure 3, we show the time taken to reach turn around. In Einstein-deSitter universe we have $t_{t a}=\pi /\left(2 H_{i n} \bar{\delta}_{i n}^{3 / 2}\right)$, implying that a perturbation with a larger initial over-density takes less time to reach turn around. We have plotted $2 H_{i n} t_{t a} \bar{\delta}_{i n}^{3 / 2} / \pi$ as a function of $\bar{\delta}_{i n} / \delta_{t h r}$. We find that this also approaches unity in the limit $\bar{\delta}_{i n} / \delta_{t h r} \gg 1$. However, we see that the combination becomes large and diverges as we approach $\bar{\delta}_{i n} / \delta_{t h r}=1$ from above. This
Figure 2. The combination $R_{T} \delta_{\text {in }} / R_{\text {in }}$ is plotted as a function of $\bar{\delta}_{i n} / \delta_{t h r}$. We choose this combination as its expected value is unity in the Einstein-deSitter model. We see that the turn around radius is larger in presence of the cosmological constant and its value increases as the initial density contrast $\bar{\delta}_{\text {in }}$ approaches $\delta_{t h r}$ from above. 
Figure 3. It takes more time for less dense (smaller $\left.\bar{\delta}_{\text {in }} / \delta_{t h r}\right)$ perturbations to reach the maximum radius (turn around) and hence by the time it reaches turn around, the background density has decreased more and hence we see a higher density contrast at turn around for these smaller initial densities (see previous figure).

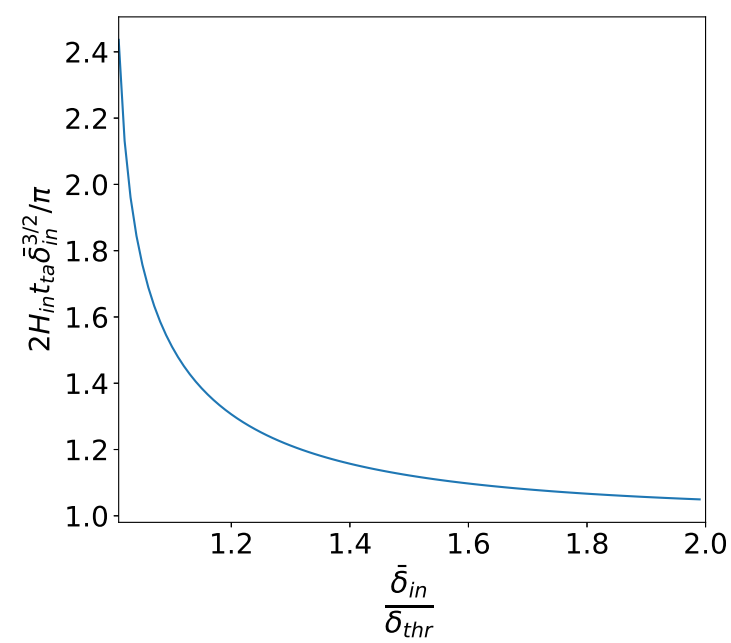

indicates that for perturbations near the critical threshold, time taken to reach turn around is significantly larger than the corresponding perturbations in the Einstein-deSitter universe. Lower panels in Figure 1 also illustrate this point.

Lastly, we plot the density contrast at turn around, $\bar{\delta}_{t a}$, as a function of $\bar{\delta}_{i n} / \delta_{t h r}$. This is shown in Figure 4. In the reference model, i.e., the Einstein-deSitter model, this has the value $9 \pi^{2} / 16-1 \simeq$ 4.55. We find that the density contrast at turn around in the case of a universe with the cosmological constant is larger and the value diverges as we approach $\bar{\delta}_{i n} / \delta_{t h r}=1$ from above. We have already noted above that the density at turn around is smaller, and we can reconcile these statements by noting that as perturbations take longer to reach turn around in a universe dominated by a cosmological constant, the average density of the Universe drops to lower values by the time the perturbation reaches turn around. Thus we have a larger density contrast or over-density at turn around, but smaller density of matter in the perturbation! 


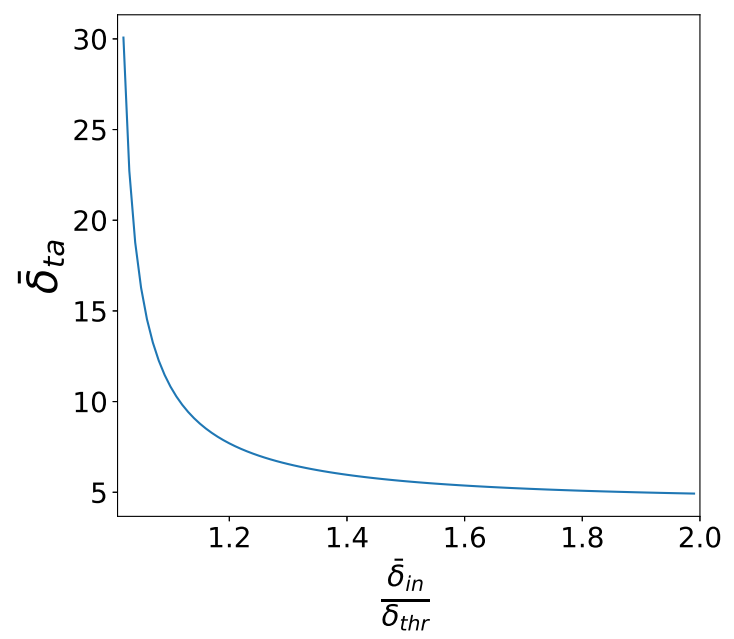

5. Virialization

Solutions for shells in cases where we have eventual collapse are oscillatory in nature, as illustrated in Figure 1. With such a solution, we may expect each shell to expand, turn around and then collapse to the origin before bouncing again. Such a fate is avoided in a real physical system where small non-radial motions arising from a small initial velocity dispersion are amplified during collapse and rapidly lead to an equilibrium state within the time it would have taken the shell to collapse back to the origin. The dynamical equilibrium is achieved when the system virializes. Radius of the system in equilibrium can be calculated using the virial theorem ${ }^{7}$.

$$
\begin{aligned}
\left\langle 2 T+\vec{F} \cdot \vec{r}_{v i r}\right\rangle & =0 \\
\text { where } \vec{F} & =-\vec{\nabla} U \\
\therefore\langle 2 T-\vec{r} \cdot \vec{\nabla} U\rangle & =0
\end{aligned}
$$

where $T$ is kinetic energy and $U$ is potential energy. In presence of the cosmological constant, there are two contributions to the potential energy and we need to take both into account. Further, given that we are discussing a single shell, we may drop the averaging to avoid confusion. For matter, gravitational potential is
Figure 4. Density contrast at turn around is plotted as a function of $\bar{\delta}_{i n} / \delta_{t h r}$. The expected value of density contrast at turn around in the Einstein-deSitter model is 4.55. We see that the density contrast at turn around in presence of cosmological constant is higher, and increases sharply as $\bar{\delta}_{i n} / \delta_{t h r}$ approaches unity from above. The perturbations are larger at turn around when the cosmological constant is present but the density contrast is higher as it takes longer to reach turn around, and the average density of the universe decreases by a large factor in this time.

\footnotetext{
${ }^{7}$ Dynamical equilibrium is achieved for a bound system when the average motions of particles in the system counter-balance the interaction forces. The motions of particles are characterized by the average kinetic energy and the interaction forces are characterized in terms of the average potential energy. Given the form of interaction, the virial theorem provides a relation to be satisfied in dynamical equilibrium. 
proportional to $r^{-1}$, and for $\Lambda$ it is proportional to $r^{2}$. Therefore,

$$
\begin{aligned}
& (\vec{r} . \vec{\nabla}) U_{m}=-U_{m} \\
& (\vec{r} . \vec{\nabla}) U_{\Lambda}=2 U_{\Lambda}
\end{aligned}
$$

Thus, we find that at virial equilibrium:

$2 T+U_{m}-2 U_{\Lambda}=2\left(T+U_{m}+U_{\Lambda}\right)-U_{m}-4 U_{\Lambda}=2 E-U_{m}-4 U_{\Lambda}=0$

The expression in brackets is the constant we evaluated in equation (7). We may evaluate it at turn around as at that stage the kinetic energy vanishes.

We use this relationship to determine virial radius in $\Lambda$ CDM model. Since at turn around the kinetic energy term is zero and because net energy is conserved, we have at Virial radius:

$$
\begin{gathered}
E=-\frac{G M}{R_{t a}}-\frac{1}{6} \Lambda R_{t a}^{2} \\
\Longrightarrow \quad-2 \frac{G M}{R_{t a}}-\frac{1}{3} \Lambda R_{t a}^{2}+\frac{G M}{R_{V}}+\frac{2}{3} \Lambda R_{V}^{2}=0 \\
\Longrightarrow \quad 2 \frac{G M}{R_{t a}}\left(1-\frac{R_{t a}}{2 R_{V}}\right)=-\frac{1}{3} \Lambda R_{t a}^{2}\left(1-2\left(\frac{R_{V}}{R_{t a}}\right)^{2}\right)
\end{gathered}
$$

It is clear that in the case of $\Lambda=0$, we get $R_{V}=R_{t a} / 2$. In the general case, substituting for $M$ and $\Lambda$, we get:

$$
\begin{aligned}
R_{V}= & \left(\frac{2}{3}\right)^{1 / 2}\left(\frac{\Omega_{\Lambda} R_{t a}^{3}+\Omega_{M}\left(\frac{a_{0}}{a_{i n}}\right)^{3}\left(1+\bar{\delta}_{i n}\right) R_{i n}^{3}}{\Omega_{\Lambda} R_{t a}}\right)^{1 / 2} \\
& \sin \left[\frac{1}{3} \arcsin \left\{\frac{\Omega_{M} a_{0}^{3}\left(1+\bar{\delta}_{i n}\right) R_{i n}^{3}}{a_{i n}^{3} R_{t a}^{3}}\left(\frac{1.5}{1+\frac{\Omega_{M}}{\Omega_{\Lambda}}\left(\frac{a_{0} R_{i n}}{a_{i n} R_{t a}}\right)^{3}\left(1+\bar{\delta}_{\text {in }}\right)}\right)^{3 / 2}\right\}\right]
\end{aligned}
$$

Figure 5 shows $R_{V} / R_{t a}$ as a function of $\bar{\delta}_{i n} / \delta_{t h r}$. It can be seen that as we go towards larger initial density contrast, the ratio of virial radius to the turn around radius approaches 0.5 from below. We see in Figure 2 that the turn around radius $R_{t a}$ is larger in presence of dark energy. Combining these, we infer that the virial radius in models with dark energy is higher as compared to models without any dark energy. The density of collapsed halos is lower as 


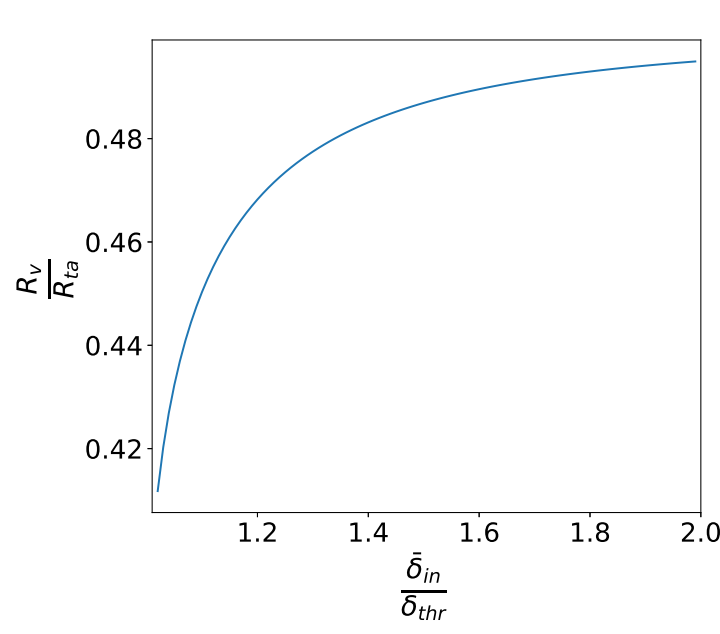

a result, though the density contrast is higher as collapse takes longer and the density of the background falls to lower values in this time.

The density contrast at virialization is shown in Figure 6, again as a function of $\bar{\delta}_{i n} / \delta_{t h r}$. We find that the density contrast is very high for perturbations where $\bar{\delta}_{i n} / \delta_{t h r}$ is just over unity. This is primarily because these perturbations take a very long time to reach virialization and the Universe expands by a larger factor in this time. This is illustrated in Figure 7. Here we plot the scale factor at virialization time $\left(a_{V} / a_{0}\right)$ for different initial perturbations. Here, $a_{0}$ is the present value of the scale factor. Time of virialization $\left(t_{V}\right)$ is generally taken to be $2 t_{t a}$ which is the exact time for analytical solution (of Einstein-deSitter) to shrink to zero, i.e., the crossing time. This continues to be approximately true in presence of the cosmological model.

\section{Discussion}

We find that the presence of cosmological constant leads to introduction of a threshold density contrast: perturbations with a smaller density contrast do not stop expanding and hence never collapse. The regions are over-dense, remain over-dense with
Figure 5. The ratio of virial radius to the turn around radius is shown as a function of $\bar{\delta}_{i n} / \delta_{t h r}$. As $\bar{\delta}_{i n} / \delta_{t h r} \rightarrow \infty$ the curve tends to EinsteindeSitter limit of 0.5 . 
Figure 6. Density contrast at virialization $\bar{\delta}_{V}$ as a function of $\bar{\delta}_{i n} / \delta_{t h r}$. Density contrast is very high for $\bar{\delta}_{i n} / \delta_{t h r}$ close to unity and it decreases as we get to larger initial density contrast, asymptotically approaching the expected value for Einstein-deSitter model as $\bar{\delta}_{i n} / \delta_{t h r} \rightarrow \infty$.

Figure 7. We have plotted the scale factor at the time of virialization $a_{V}$ as a function of $\bar{\delta}_{i n} / \delta_{t h r}$. It takes more time for perturbations with a smaller initial density contrast $\left(\bar{\delta}_{i n} / \delta_{t h r}\right)$ to virialize and hence by the time it virializes, the background density has decreased more and hence we see a higher virial density contrast for these smaller densities(see previous figure).
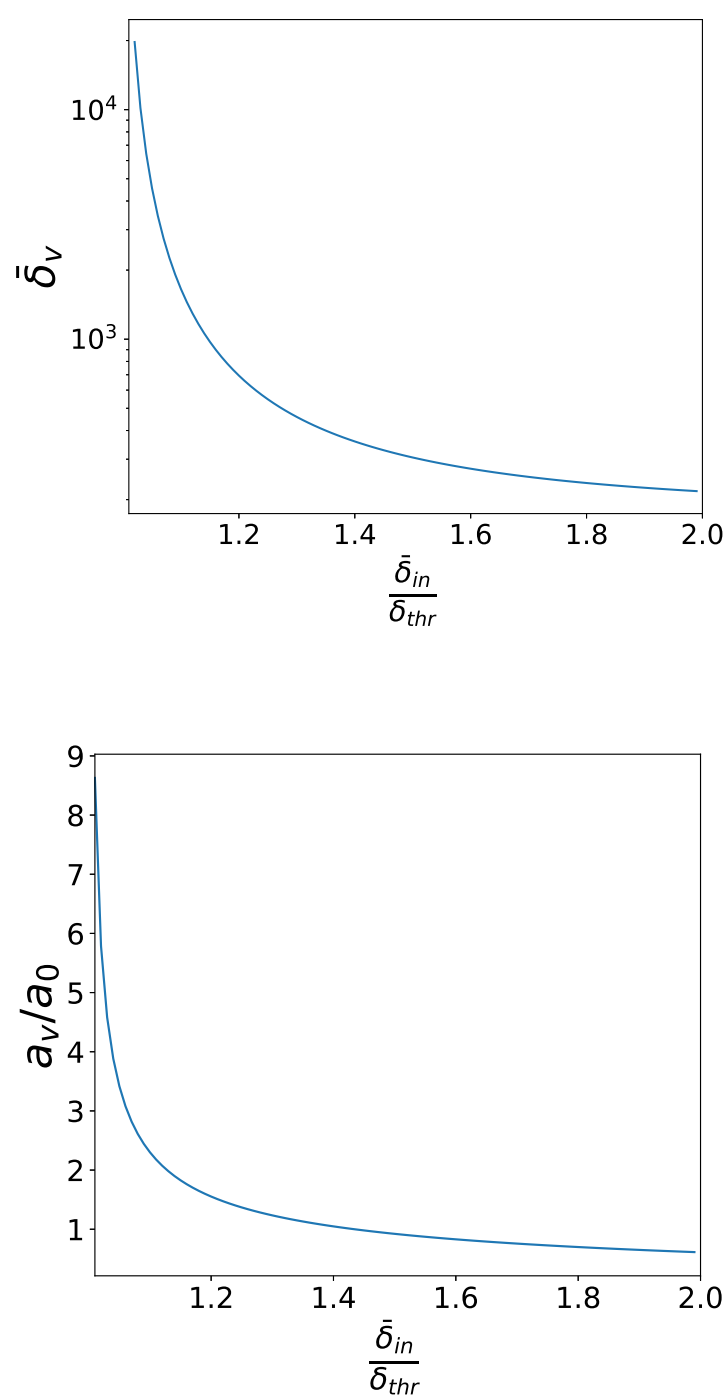

a steadily increasing density contrast that never collapse. The threshold density contrast is independent of the scale of perturbation.

It takes longer to form collapsed structures, hence the process of structure formation must start with higher initial density contrast as compared to the Einstein-deSitter universe. Thus overdensities must have a higher value at early times. For example, 
we expect to see more clusters of galaxies at high redshifts in such a universe.

These findings have observable implications for galaxy formation and the formation of large scales structure in the Universe.

\section{Acknowledgements}

The authors thank the anonymous referee for a very detailed reading and helpful comments.

\section{Suggested Reading}

[1] Callahan J. J., SciAm, 235, 90, 1976

[2] Einstein, A., Sitzungsberichte der Königlich Preußischen Akademie der Wissenschaften (Berlin), Seite 142-152., 142, 1917

[3] O'Raifeartaigh et al, "Einstein's 1917 static model of the universe: a centennial review" arXiv:1701.07261 v2, 2017 https://blogs.scientificamerican.com/guest-blog/einsteins-greatest-blunder/

[4] Friedmann A., Z.Phys. 10, 377, 1922

[5] Lemaître G., Ann.Soc.Sci.Bruxelle, 47A, 49, 1927

[6] Robertson H.P., Phil.Mag. 5, 835, 1928

[7] Belenkiy, A., arXiv:1302.1498 "The Waters I am Entering No One yet Has Crossed": Alexander Friedman and the Origins of Modern Cosmology, 2013

[8] Lemaître, G., Monthly Notices of the Royal Astronomical Society, Vol. 91, p.483-490, 1931

[9] Robertson, H. P., Astrophysical Journal, vol. 82, p.284, 1935

[10] Walker A. G., QJMat, 6, 81, 1935

[11] Slipher, V. M., Popular Astronomy, Vol. 23, p. 21-24, 1915

[12] Hubble, Edwin, "A Relation between Distance and Radial Velocity among Extra-Galactic Nebulae" Proceedings of the National Academy of Sciences of the United States of America, Volume 15, Issue 3, pp. 168-173, 1929

[13] Baade W., PASP, 68, 5, 1956

[14] J. S. Bagla, Resonance 14, 216, 2009

[15] S. Perlmutter, G. Aldering, M. della Valle, et al., Nature 391, 51, 1998

[16] A. G. Riess, A. V. Filippenko, P. Challis, et al., AJ 116, 1009, 1998

[17] B. P. Schmidt, N. B. Suntzeff, M. M. Phillips, et al., ApJ 507, 46, 1998

[18] J. E. Gunn \& J. R. Gott III, ApJ 176, 1, 1972

[19] J. S. Bagla \& Pritpal Kaur Sandhu, Resonance 20, 803, 2015

[20] Planck Collaboration, et al., arXiv:1807.06209 2018

[21] Peebles P. J. E., ApJ, 284, 439, 1984

[22] Lahav O., Lilje P. B., Primack J. R., Rees M. J., MNRAS, 251, 128, 1991

[23] John D. Barrow \& Paul Saich, MNRAS 262, 717, 1993

[24] Eke V. R., Cole S., Frenk C. S., MNRAS, 282, 1996 